\title{
Quark mass dependence of light resonances and phase shifts in elastic $\pi \pi$ and $\pi K$ scattering
}

\author{
J. Nebreda and J.R. Peláez \\ Dept. Física Teórica II. Universidad Complutense, 28040, Madrid. Spain
}

\begin{abstract}
We study the light quark mass dependence of the $\pi \pi$ scattering phase shifts in standard one and two-loop SU(2) Chiral Perturbation Theory (ChPT). We then repeat the study with unitarized ChPT and, furthermore, we extend the analysis to SU(3) and generate the elastic $f_{0}(600)$, $\kappa(800), \rho(770)$ and $K^{*}(892)$ resonances from unitarization. The quark masses are varied up to values of interest for lattice studies. We find that the $\mathrm{SU}(2) \pi \pi$ phase shifts both in standard and unitarized ChPT depend very softly on the pion mass and that our results are in fair agreement with lattice results in the $\mathrm{I}=2, \mathrm{~J}=0$ channel. In the $\mathrm{SU}(3)$ amplitudes, the mass and width of the $\rho(770)$ and $K^{*}(892)$ present an analogous and smooth quark mass dependence. In contrast, both scalars present a similar non-analyticity at high quark masses. We also confirm the lattice assumption of independence of the vector two-meson coupling on the quark mass, that is, nevertheless, violated for scalars.
\end{abstract}

PACS: $14.40 . \mathrm{Cs}, 12.39 . \mathrm{Fe}, 13.75 . \mathrm{Lb}$

\section{INTRODUCTION}

Although QCD is well established as the theory of strong interactions, the hadronic realm is beyond the reach of perturbative calculations. In that regime, lattice methods are a useful tool to calculate QCD observables, but results on light meson resonances are few and usually obtained at very large quark masses compared with their physical values. On the other hand, Chiral Perturbation Theory (ChPT) provides the quark mass dependence of the meson-meson scattering amplitudes in the low energy region. Thus, we are able to compare its results to those of lattice studies by increasing the mass up to the applicability limits of our theory. In this talk we first study the phase shift dependence on the averaged $u$ and $d$ quark mass, $\hat{m}$, in standard one and two-loop SU(2) ChPT[1]. This approach has the advantage of being completely model independent but it is limited to the low energy region and can not generate resonances. This is the reason why we next extend our study to unitarized ChPT, using the well-known one-loop elastic Inverse Amplitude Method (IAM), which allows us not only to calculate the phase shift quark mass dependence up to higher values of $\hat{m}$, but also to generate poles on the second Riemann sheet and study their dependence on the quark masses. This technique was applied in [2] to calculate the $\hat{m}$ dependence of the $f_{0}(600)$ (or "sigma") and $\rho(770)$ resonances and then we extended this study in [3] to include the strange quark within a unitarized SU(3) ChPT formalism, so that we could also generate the $K^{*}(892)$ and $\kappa(800)$ resonances and vary both the light and the strange quark masses.

CP1322, International Workshop on Chiral Symmetry in Hadrons and Nuclei edited by J. M. Nieves, E. Oset, and M. J. Vicente Vacas

(C) 2010 American Institute of Physics 978-0-7354-0878-4/10/\$30.00 

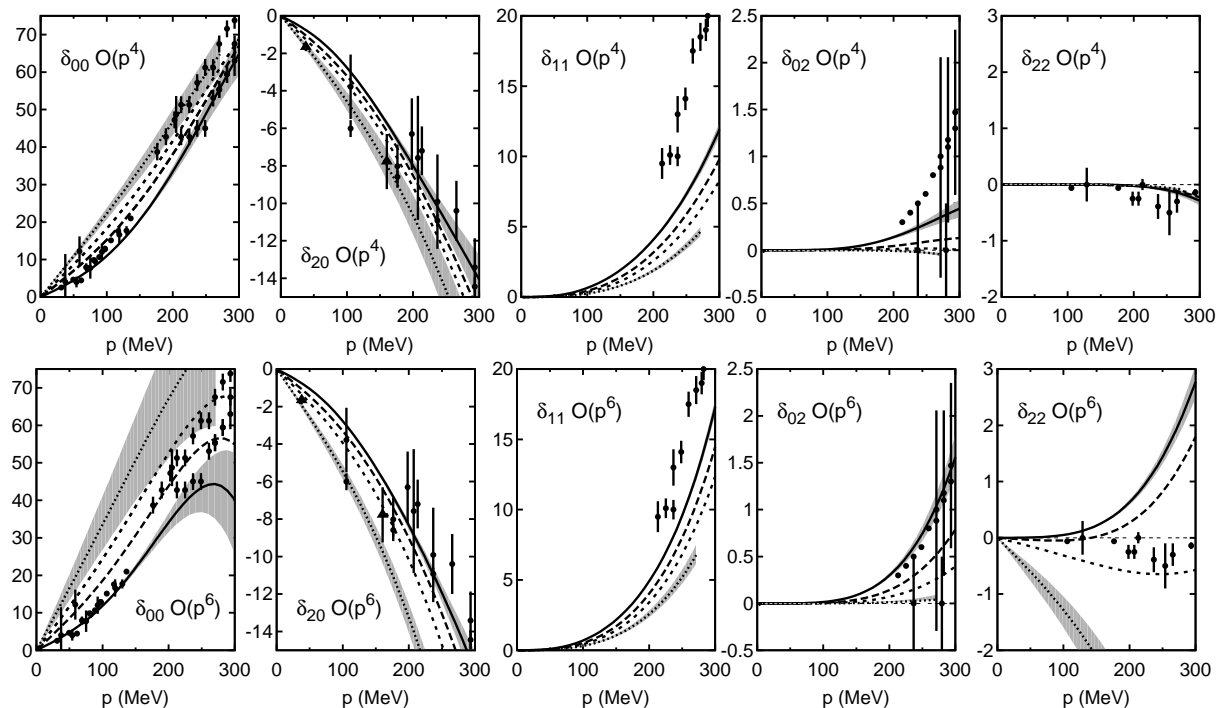

FIGURE 1. $\pi \pi$ phase shifts from standard ChPT up to one loop (first row) and to two loops (second row). Different lines stand for different pion masses: continuous, long dashed, short dashed and dotted for $M_{\pi}=139.57,230,300$ and $420 \mathrm{MeV}$ respectively. Because of applicability considerations, we do not plot phase shifts beyond $\sqrt{s}=1 \mathrm{GeV}$. Since the lines are too close to each other, we only show error bands for the lightest and heaviest masses. Experimental data (circles) come from [5]. Lattice results for the I=2, $\mathrm{J}=0$ channel (triangles) come from [6].

\section{QUARK MASS DEPENDENCE IN STANDARD CHPT}

We show preliminary results for the dependence of the $\pi \pi$ phase shifts on $\hat{m}$ using the SU(2) scattering amplitudes in [1] and the LECs in [4]. In Fig.1 we show phases on different channels for different pion masses, to one and two loops (first and second row respectively). They are plotted as a function of the center of mass momentum -and not of the energy- in order to subtract the shift of the thresholds. Since the applicability of plain ChPT is limited to low energies, we do not plot phase shifts beyond $\sqrt{s}=1 \mathrm{GeV}$. In order to compare with lattice results [6], we rise $M_{\pi}$ up to $420 \mathrm{MeV}$, although most likely the IAM is not reliable in that region. A Montecarlo gaussian sampling based on the errors of the LECs has been used to calculate the error bands. We find that the dependence of the phase shifts on $\hat{m}$ is very soft at one loop and somehow stronger at two loops, specially for the $\mathrm{I}=2, \mathrm{~J}=2$ channel, and that the results are compatible with those from lattice in the $\mathrm{I}=2, \mathrm{~J}=0$ channel [6].

\section{QUARK MASS DEPENDENCE IN UNITARIZED CHPT}

We use now the IAM to unitarize our amplitudes. This procedure extends their applicability up to the resonance region and generates poles in the second Riemann sheet, which relate to resonances through the usual Breit-Wigner identification $\sqrt{s_{\text {pole }}} \equiv M-i \Gamma / 2$. Furthermore, the coupling of the resonance to two mesons is given by the residue of the amplitude at the pole position. 

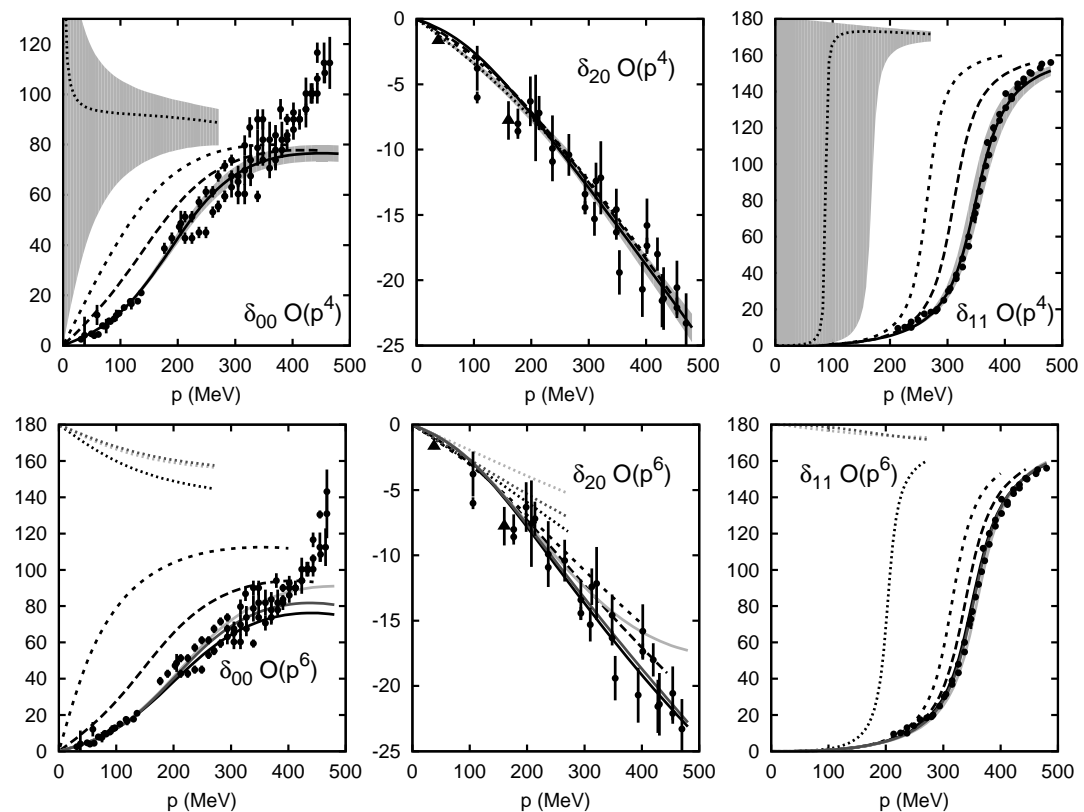

FIGURE 2. $\pi \pi$ phase shifts from unitarized ChPT up to one loop (first row) and to two loops (second row). Different lines stand for different pion masses: continuous, long dashed, short dashed and dotted for $M_{\pi}=139.57,230,300$ and $420 \mathrm{MeV}$ respectively. Because of applicability considerations we do not plot phase shifts beyond $\sqrt{s}=1 \mathrm{GeV}$. For the lightest and heaviest masses we show results for three different sets of LECs [7] (black, dark gray and light gray) in order to give an idea of the size of the errors. Experimental data (rounded points) come from [5]. Lattice results for the $\mathrm{I}=2 \mathrm{~J}=0$ channel come from [6].

Phase shifts. In Fig. 2 we show preliminary $\pi \pi$ phase shifts (note that the one and two-loop IAM cannot be used for the D-waves) for different pion masses. For the one loop analysis (first row) we used the LECs in [2] and found that the dependence is again quite soft, specially for the $\mathrm{I}=2, \mathrm{~J}=0$ channel. For the two loop analysis (second row) we used some preliminary sets of LECs [7]. Once more the dependence on the pion mass is stronger at two loops than at one loop.

Resonance dependence on the light quark mass [3]. The vector resonances are well established $q \bar{q}$ states belonging to an SU(3) octet. In the two upper rows of Fig. 3 we show the dependence of $\rho(770)$ and $K^{*}(892)$ on the light quark mass. The results obtained for $\rho(700)$ are very consistent with the SU(2) analysis [2] (dotted line) and the estimations for the two first coefficients of the $M_{\rho}$ chiral expansion [8]. $\rho(770)$ and $K^{*}(892)$ behave very similarly: their masses increase smoothly as the quark mass increases, but much slower than $M_{\pi}$. As a consequence there is a strong phase space suppression which accounts by itself for the width decrease, without a dynamical effect through the couplings $g_{\rho \pi \pi}$ and $g_{K^{*} \pi K}$, that are remarkably constant, which is an assumption made in lattice studies of the $\rho(770)$ width [9].

On the other hand, the $f_{0}(600)$, or sigma, and the $\kappa(800)$ scalar mesons are still somewhat controversial since their huge width makes their experimental identification com- 

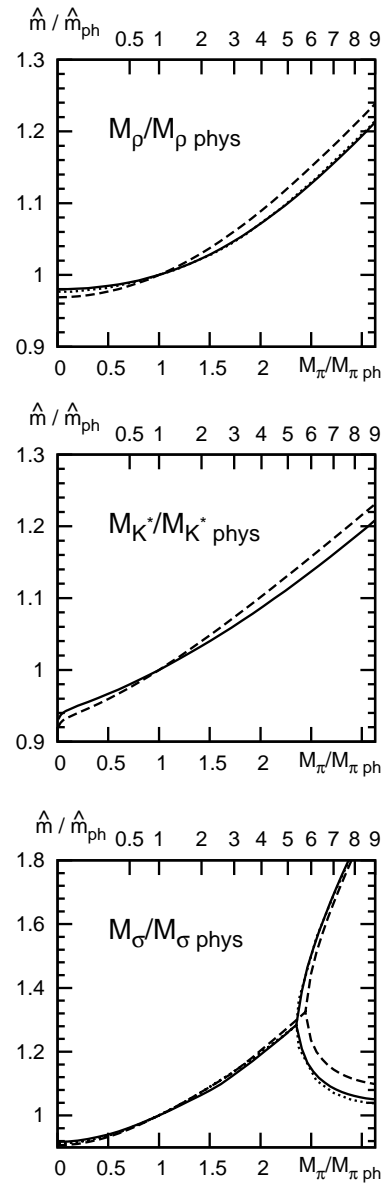

$\hat{\mathrm{m}} / \hat{\mathrm{m}}_{\mathrm{ph}} 0.51 \quad 2 \quad 34456789$

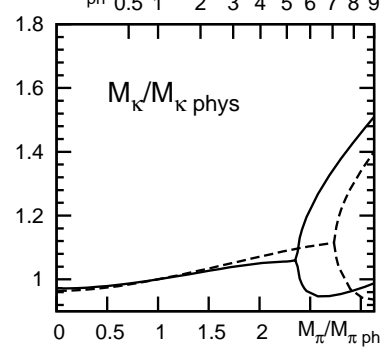

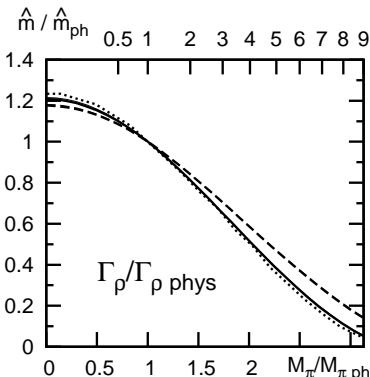
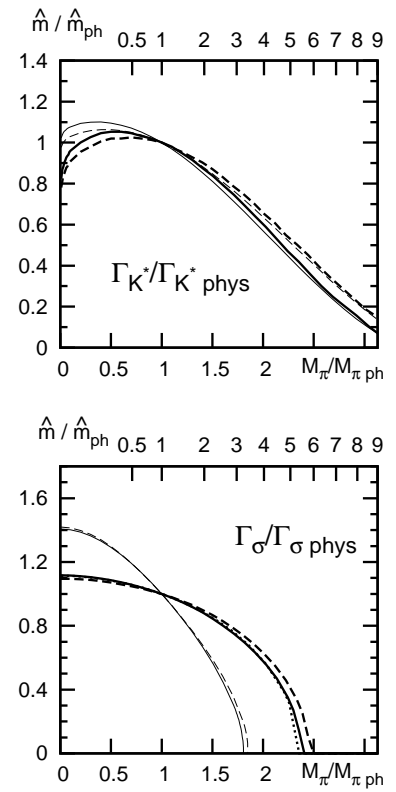

$\hat{\mathrm{m}} / \hat{\mathrm{m}}_{\mathrm{ph}} 0.51 \quad 2 \quad 3 \quad 4 \quad 56789$

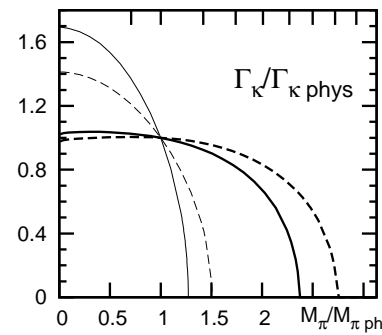

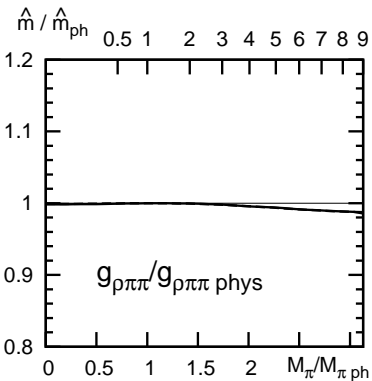

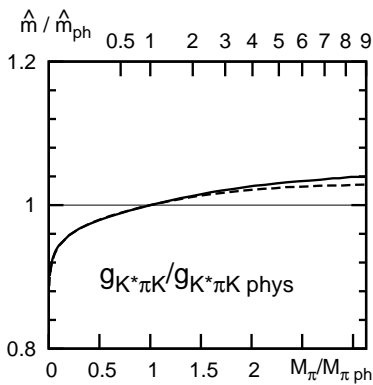

$\hat{\mathrm{m}} / \hat{\mathrm{m}}_{\mathrm{ph}} 0.51 \quad 2 \quad 3 \quad 4 \quad 5 \quad 6 \quad 7 \quad 89$

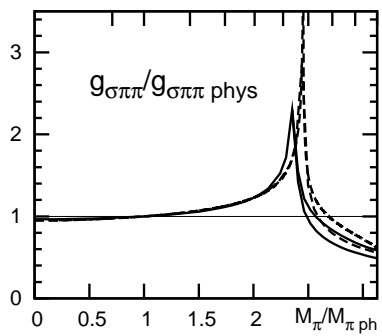

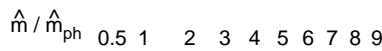

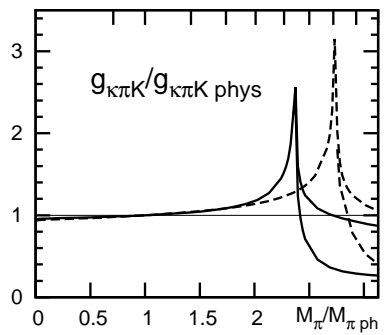

FIGURE 3. Dependence of the $\rho(770), K^{*}(892), f_{0}(600)$ and $\kappa(800)$ mass, width and coupling to two mesons with respect to $\hat{m}$ (horizontal upper scale) or $M_{\pi}$ (horizontal lower scale). Note that all quantities are normalized to their physical values. The thick continuous and dashed lines correspond to fits I and II in Ref. [3], respectively. The $\rho(770)$ and $f_{0}(600)$ dependence on the light quark mass is very compatible with that in [2] (dotted lines). Continuous (dashed) thin lines show the dependence of the widths from the change of phase space only, assuming a constant coupling of the resonances to two mesons, using Fit I (II). 

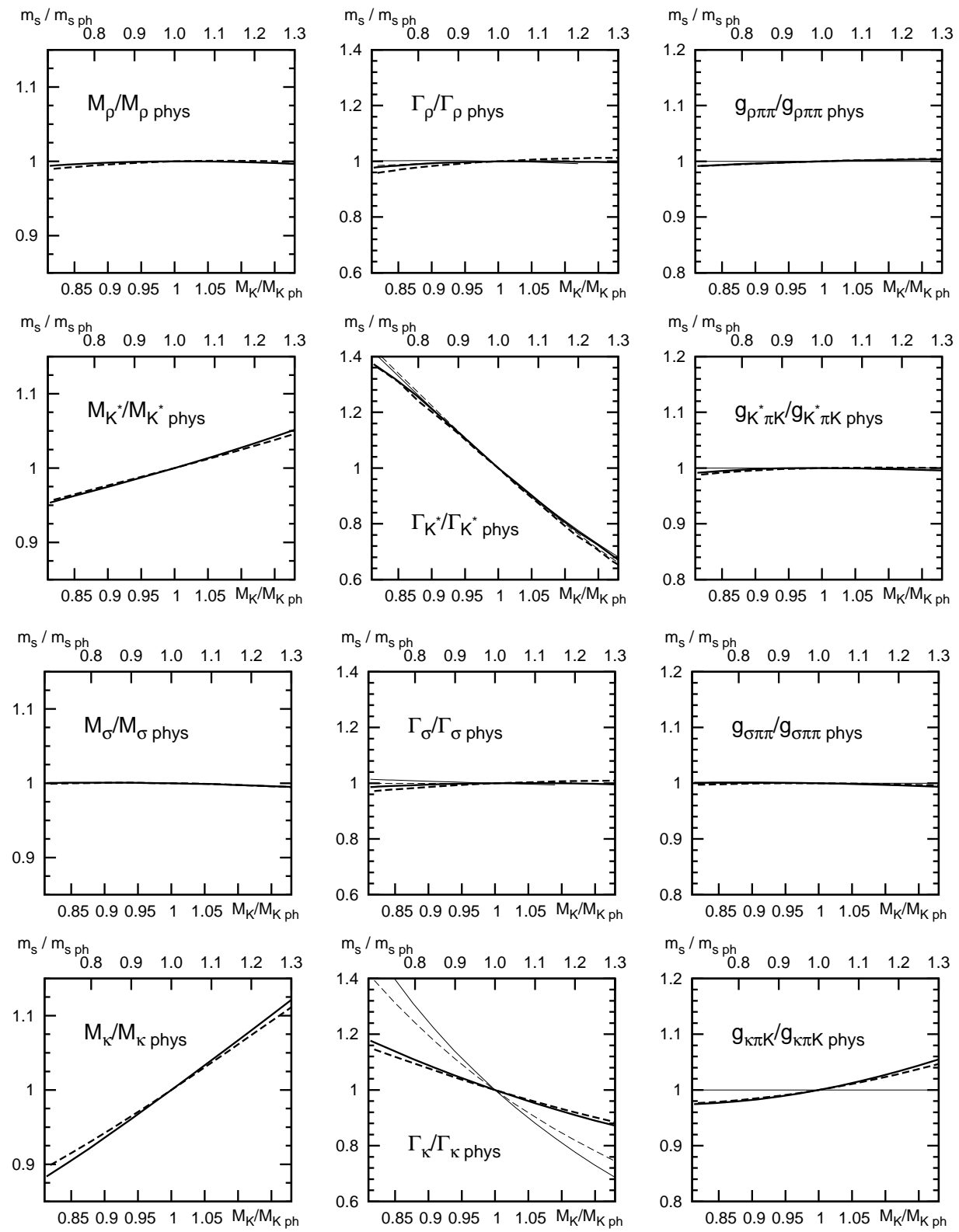

FIGURE 4. Dependence of the $\rho(770), K^{*}(892), f_{0}(600)$ and $\kappa(800)$ mass, width and coupling to two mesons with respect to $m_{s}$ (horizontal upper scale) or $M_{K}$ (horizontal lower scale). Note that all quantities are normalized to their physical values. The thick continuous and dashed lines correspond to fits I and II in Ref. [3], respectively. Continuous (dashed) thin lines show the dependence of the widths from the change of phase space only, assuming a constant coupling of the resonances to two mesons, using Fit I (II). 
plicated. In the two lower rows of Fig. 3 we show their dependence on $\hat{m}$. As before, the $f_{0}(600)$ the results are in good agreement with [2]. Their most prominent feature is the splitting of the mass into two branches when the associated conjugated poles in the second Riemann sheet, that are getting closer to the real axis as the quark mass increases, join in a single pole below threshold and then split again and remain in the real axis. However, the quantitative behavior of the $f_{0}(600)$ and the kappa is rather different. In particular, the growth of the $f_{0}(600)$ mass before the "splitting point" is much faster than that of the $\kappa(800)$. Regarding their width decrease, we show that for the scalars it cannot be attributed to the phase space reduction due to the increase of pion and kaon masses, since their coupling to two mesons show a strong quark mass dependence.

Resonance dependence on the strange quark mass [3]. We will only vary the strange quark mass in the limited range $0.8<m_{s} / m_{s \text { phys }}<1.4$ to ensure that the kaon does not become too heavy to spoil the ChPT convergence nor too light to require a coupled channel formalism. In Fig. 4 we show the kaon (or strange quark) mass dependence of the $\rho, K^{*}(892)$, sigma and $\kappa(800)$. As it could be expected, the properties of the $\rho(770)$ and sigma non-strange resonances are almost independent of the strange quark mass within the range of study. Obviously, the $K^{*}(892)$ and the $\kappa(800)$ show a strong $m_{s}$ dependence. As the kaon mass is made heavier, their masses grow much faster than they did when increasing the light quark mass, but still much slower than the kaon mass. As before, in the case of the vector resonance the width decreases almost exactly as expected from phase space suppression only and its coupling to $K \pi$ is almost constant, while the scalar width decrease deviates significantly from that behavior, in agreement with $g_{\kappa \pi K}$ depending quite strongly on the strange quark mass.

\section{ACKNOWLEDGMENTS}

Work partially supported by Spanish MICINN: FPA2007-29115-E, FPA2008-00592 and FIS2006-03438, U.Complutense/ Banco Santander grant PR34/07-15875-BSCH and UCM-BSCH GR58/08 910309 and the EU-Research Infrastructure Integrating Activity "Study of Strongly Interacting Matter" (HadronPhysics2, Grant n 227431) under the EU Seventh Framework Programme.

\section{REFERENCES}

1. J. Bijnens, G. Colangelo, G. Ecker, J. Gasser and M. E. Sainio, Phys. Lett. B 374, 210 (1996).

2. C. Hanhart, J. R. Pelaez and G. Rios, Phys. Rev. Lett. 100, 152001 (2008).

3. J. Nebreda and J. R. Pelaez., Phys. Rev. D 81, 054035 (2010).

4. G. Colangelo, J. Gasser and H. Leutwyler, Nucl. Phys. B 603, 125 (2001).

5. L. Rosselet et al., Phys. Rev. D 15, 574 (1977). S. Pislak et al. [BNL-E865 Collaboration], Phys. Rev. Lett. 87, 221801 (2001) G. Grayer et al., Nucl. Phys. B 75, 189 (1974). S. D. Protopopescu et al., Phys. Rev. D 7, 1279 (1973). W. Hoogland et al., Nucl. Phys. B 126, 109 (1977). M. J. Losty et al., Nucl. Phys. B 69, 185 (1974). N. B. Durusoy et al., Phys. Lett. B 45, 517 (1973). P. Estabrooks and A. D. Martin, Nucl. Phys. B 79, 301 (1974). B. Hyams et al., Nucl. Phys. B 64, 134 (1973). D. H. Cohen, T. Ferbel, P. Slattery and B. Werner, Phys. Rev. D 7, 661 (1973). J. R. Batley et al. [NA48/2 Collaboration], Eur. Phys. J. C 54, 411 (2008).

6. K. Sasaki and N. Ishizuka, Phys. Rev. D 78, 014511 (2008).

7. J. R. Pelaez and G. Rios, in preparation.

8. P. C. Bruns and U.-G. Meißner, Eur. Phys. J. C 40 (2005) 97.

9. S. Aoki et al. [CP-PACS Collaboration], Phys. Rev. D 76, 094506 (2007). 
Copyright of AIP Conference Proceedings is the property of American Institute of Physics and its content may not be copied or emailed to multiple sites or posted to a listserv without the copyright holder's express written permission. However, users may print, download, or email articles for individual use. 